\title{
Evaluation of the Outcome of Tibial Shaft Fracture by Close Reduction and Internal Fixation with Interlocking Intramedullary Nail
}

\author{
Abdul Khaleque ${ }^{1 *}$, Md. Mohiuddin Aslam², Md. Nazrul Islam ${ }^{3}$ \\ ${ }^{1}$ Assistant Professor (Orthopaedics), National Institute of Traumatology \& Orthopaedic Rehabilitation (NITOR), Sher E Bangla Nagar, Dhaka-1207, \\ Bangladesh \\ ${ }^{2}$ Assistant Professor (Orthopaedics), Shaheed Ziaur Rahman Medical College Hospital, Bogura, Bangladesh \\ ${ }^{3}$ Registrar (Orthopaedics), Shaheed Ziaur Rahman Medical College Hospital, Bogura, Bangladesh
}

DOI: $10.36348 /$ sjmps.2020.v06i01.011

| Received: 01.01.2020 | Accepted: 10.01.2020 | Published: 22.01.2020

*Corresponding author: Abdul Khaleque

\section{Abstract}

Background: Tibial shaft fractures were common occurrence due to road traffic accident, most casualty affecting the active working people, male were more affected than female. Treatment of diaphyseal fractures were treated by close reduction and internal fixation with interlocking intramedullary nail showed in many study with excellent results. Objective: To evaluate and analyze the results and outcome of tibial shaft fractures by interlocking intramedullary nail. Methods: This prospective observational study (case series) was conducted in the department of orthopaedic and traumatology department, Dhaka Medical College and Hospital and NITOR from January 2011 to December 2012. This study included both sexes and age between 18 years to 55 years and 30 patients were included in this study. As per inclusion and exclusion criteria all stable or non-displaced unstable or displaced and Gustilo type-1 fractures of proximal, middle and distal third fractures were included in this study .Diagnosis of the tibial diaphyseal fracture was made by history, clinical examination \& radiological evidence. Results: A total number of 30 patients were treated and followed up in this study. Among 30 patients 21 (70\%) were male and $9(30 \%)$ were female, mean age was 31.8 years with a range of 18 years to 55 years median was 32.5 years mode was 34 years and SD was 2.1. in the study mode of injury distribution was $21(70 \%)$ RTA, $6(20 \%)$ fall from height and $3(10 \%)$ assault. In this study severity injury $46.7 \%$ are stable $33 \%$ are unstable and $23.3 \%$ was Gustilo-1 , distribution of level of fracture was middle third $57 \%$ distill third $29 \%$ and proximal third $17 \%$, injury surgery interval average 11.5 days $(60 \%)$ in 18 cases and 5 days (40\%) in 12 cases. Partial weight bearing given at 2 weeks \& full weight bearing at 6 weeks and union occurred in average 18.5 weeks. In rating of final outcome 24 cases $(80 \%)$ were excellent, 3 cases $(9 \%)$ were good, 2 cases $(7 \%)$ were fair and 1 case $(3 \%)$ was poor. In final outcome categorization satisfactory were $90 \%$ and unsatisfactory were $10 \%$. Conclusion: This study permits to conclude that close interlocking intramedullary nail for treatment of tibial diaphyseal fracture is safe, with a high rate of union and early mobilization with a low complication rate and early return to function.

Keywords: Tibial shaft fractures, radiological evidence, Gustilo type-1 fractures, Internal Fixation.

Copyright @ 2020: This is an open-access article distributed under the terms of the Creative Commons Attribution license which permits unrestricted use, distribution, and reproduction in any medium for non-commercial use (NonCommercial, or CC-BY-NC) provided the original author and source are credited.

\section{INTRODUCTION}

Fractures of the tibial shaft are a common occurrence and are the most frequent fractures involving shaft of tibia due to most accessible parts and subcutaneous position. The most appropriate treatment option depends on thoughtful analysis of the morphology of fracture, the severity of injury, the mechanical characteristics of bone, the age and general condition of the patient and most importantly the status of soft tissues. Being the weight bearing bone, maximum thrust of the body passes through it. Disability following tibial shaft fractures may result in serious sequel like nonunion. The average hospital stay of the patients was five days. The patients were discharged with advice to active toe movement, quadriceps exercise and to come for suture removal after 10 to 14 post-operative day. Partial weight bearing was started with the help of two axillary crutches with toe touches. The average duration of partial weight bearing was given six weeks (range 4 to 8 weeks). The average duration of full weight bearing was given 12 weeks (range 10-14 weeks). The union of tibial shaft fracture is related to severity of injury. Other factors such as degree of fracture comminution, degree of soft tissue injuries, open fracture and medical comorbidities, patient lifestyles, tobacco use, nutritional status, and medications may play a role. Slight variation from 
normal alignment of tibial shaft is incompatible with proper function of the limb. So it is essential to obtain anatomical reduction and stabilization following such fractures [1]. It is very important in the tibial shaft fracture that a definitive diagnosis is made, based on the clinical evaluation of history, mechanism of injury, determination of the degree of immediate disability, proper examination of the injured part and obtaining proper radiograph to demonstrate the tibial shaft fracture [2]. Close reduction and internal fixation with intramedullary interlocking nail for tibial shaft fracture is popularized common method due to biological fixation with less soft tissue trauma. This method gives early weight bear less hospital stay and early return to daily normal activities. But its disadvantage is require portable x-ray facility or image intensifier like c-arm. Although closed method of reduction and immobilization has been tried years, yet the current opinion increasingly favors primary operative intervention for stable or unstable tibial shaft fractures of the lower limb [3] Denham was [4], probably the first to emphasize the importance of the perfect operative reduction. He advocated secure internal fixation for many fractures, emphasized the importance of open reduction, rigid fixation and early mobilization for all unstable tibial shaft fractures [4]. There are three reasons why unstable fractures should be treated by internal fixation: 1) They are difficult to reduce, 2) They are hard to hold reduced while uniting, and 3) If treated by conservative method, the mobilization of the joints and the treatment of the soft tissue are not easy [5].

\section{OBJECTIVES \\ General Objective}

a) To assess the success rate of treatment of 'Tibial shaft fracture by Close reduction and internal fixation with interlocking intramedullary nail".

\section{Specific Objectives}

a) To evaluate the results and outcomes with regards

b) To assess the time and rate of union of fracture of the tibial shaft.

c) To assess functional outcome of tibial shaft fractures.

d) To assess complications.

\section{Methods And Materials}

This prospective observational study (case series) was conducted in the department of orthopaedic and traumatology department, Dhaka Medical College and Hospital and NITOR from January 2011 to December 2012. This study included both sexes and age between 18 years to 55 years and 30 patients were included in this study. As per inclusion and exclusion criteria all stable or non-displaced unstable or displaced and Gustilo type-1 fractures of proximal, middle and distal third fractures were included in this study. Diagnosis of the tibial diaphyseal fracture was made by history, clinical examination \& radiological evidence.

\section{Inclusion Criteria}

- $\quad$ Age of the patient - Adult between 18 years to 55 years of age.

- Stable (minimally displaced) tibial shaft fracture

- Unstable (moderately displaced) tibial shaft fracture

- Severly displaced tibial shaft fracture [19]

\section{Exclusion Criteria}

- Patient below 18 years or above 55 years of age.

- Open fractures; Gustilo type two \& three.

- Fracture of shaft of tibia with medically unfit for surgery

- Infected case

\section{Surgical Anatomy}

The tibia is the medial and much stronger of the two bones of the leg. It is the second largest bone of the body. It is prismoid in section in the shaft and is expanded more from side and forms two large condyles which overhang the posterior surface of the shaft. The upper end of tibia includes the medial and lateral condyles, non-articular intercondylar area and the tuberosity of tibia. The lower end is smaller than the upper end and on its medial side is a stout process the medial malleolus which projects down to take part in formation of the ankle joint. The fibula is the lateral bone of the leg. It is attached to the tibia at the fibular facet on the lateral condyle and below to the fibular notch. The interosseous membrane attaches to the interosseous border of tibia and fibula except at the upper and lower end. Although the fibula does not significantly contribute to transmission of weight, its continuity helps in stabilizing unstable fractures and maintaining length in severely comminute fractures. The lower end of fibula forms the lateral malleolus and forms the lateral mortise of ankle joint and is extremely important for the structural stability of the ankle.

\section{Description of AO Tibial-Nail}

A specially constructed Interlocking Intramedullary -nail was used for the purpose in all cases. Interlocking Intramedullary nail is a hollow, metallic modified nail having a proximal end (head) and two proximal locking hole. It has proximal bent of $15^{\circ}$ in antero-posterior direction to compensate for the proximal curve within the medullary canal. About $2.5 \mathrm{~cm}$ above the tip of the nail has a distal locking hole in the coronal plane. A suitable length of the nail is chosen by measuring from the tibial tuberosity to the base of the medial malleolus on the unaffected side. 
The diameter of the nail is decided according to the size of medullary canal on the X-ray or by reaming.

\section{Operative Procedure}

All cases were operated within 15 days of the injury. All cases were done in the orthopaedic operation theatre. Close intramedullary nailing was done without opening the fracture site and with reaming.

\section{Operative Technique}

In the operation theatre, under anaesthesia, under all aseptic precaution, painting and draping done. Then a tourniquet is applied and a pad is placed under the proximal part of the thigh. A 3" long incision is marked on the anteromedial aspect of the tibial tuberosity. The periosteum is incised along the skin incision

\section{Post-Operative Regimen}

The limb was kept elevated at all times and active toe movements were encouraged. The patient was watched for excessive swelling, pain and distal circulation. The first dressing was done after 5 days of the operation. If suture line was clean, suture removal done after 10 to 12 days under full asepsis. The compression bandage was removed and a crepe bandage was applied from knee to the ankle. Active knee and ankle mobilization was started immediately after the dressing. Partial weight bearing with axillary crutches started and reviewed after one month. Advice regarding full weight -bearing was given on the basis of pain and the stability of the fracture fixation.

\section{Follow-up and Evaluation}

The patient was usually followed up at 4 weeks, 8 weeks, and 12 weeks, 16 weeks, 20 weeks, and 36 weeks. Check X-rays were taken at every visit and patient was assessed clinically for fracture union.

\section{First visit after 2 weeks}

- Long leg back slab was discarded after 2 weeks. Stitches were removed.

- Operation site was observed for evidence of swelling and infection.

- $\quad$ Range of movement of the knee and ankle was recorded

- $\quad$ Physiotherapy for joint movement and muscle strength (straight leg raising) was advised.

- Check X-ray of the leg including knee and ankle joints in antero-posterior and lateral views was taken to see bony alignment and position of nail and screws.

- Toe touch walking with double crutches was initiated.

\section{Second visit after 6 weeks}

- Check x-ray was taken to see bony alignment, call us at the fractures site and position of nail and screws.
- Operation site was observed for evidence of swelling and infection.

- $\quad$ Range of motion of knee and ankle joint was assessed.

- Physiotherapy was given to increase muscle strength and range of movement.

- When progression of union was seen clinically and radiologically then partial weight bearing was given with double crutches.

After 3 Months

- Patient was assessed for

- Pain on full weight bearing and kneeling

- Operation site was observed

- Shortening

- Range of motion of knee and ankle

- Radiological assessment for:

- Union of fracture

- Anatomical alignment of fracture and angulations

- Position of nail and screws

- Infection

\section{After 1 year}

- Patient was assessed for

- Pain on full weight bearing and kneeling

- Shortening

- Range of motion of knee and ankle joints

Patient was followed up for 3 monthly intervals for 18 months. The functional ability in the form of pain on full weight bearing and kneeling, amount of shortening, range of motion of knee and ankle joint, alignment and angulations of fracture at healing, fracture healing without infection, were expressed as excellent, good, fair and poor according to the criteria [6].

\section{Utilization of the Result}

The result of this study will make the orthopaedic surgeons confident about the technical method of treatment of unstable fracture of the proximal, middle and distal third of the tibial shaft in adults.

\section{Facilities Required}

Existing facilities of DMCH and NITOR was suitable as image intensifier (C-arm) facilities were available here. Required implants were also available with cheap prices.

\section{Ethical implication}

Informed written consent was taken from the patient or patient's guardian after duly informing the procedure of treatment, anticipated result, possible advantages, disadvantages and complications considering all ethical issues. Confidentiality was 
maintained both verbally and documentarily by using separate locker and computer password.

\section{RESULTS}

This prospective observational study was conducted in the department of Orthopaedics \& Traumatology, Dhaka Medical College and Hospital and NITOR, Dhaka from July 2011 to December 2012 for the duration of 18 months. All patients with clinical and radiological evidence of stable and unstable tibial shaft fracture admitted in this hospital age between 18 years to 55 years in both sexes were included in this study. A total number of 30 patients were enrolled in this study as per inclusion and exclusion criteria. Diagnosis of the tibial shaft fracture was made by history, clinical examination and radiological evidence. Patients were treated with closed reduction and internal fixation by interlocking intramedullary nail. Patients were followed up regularly for thirty-six weeks.

Table-1: Distribution of the patients by age $(\mathrm{N}=30)$

\begin{tabular}{|l|l|l|}
\hline Age in years & Frequency & Percentage \\
\hline $18-24$ & 6 & 20.0 \\
\hline $25-34$ & 16 & 53.3 \\
\hline $35-44$ & 5 & 16.7 \\
\hline $45-55$ & 3 & 10.0 \\
\hline Total & 30 & 100.0 \\
\hline & & Range \\
\hline & & $18-55$ \\
\hline
\end{tabular}

Age distribution of the total 30 patients was between 18 years to 55 years, mean was 31.8 years (Table-1).

\section{Distribution of patients by sex}

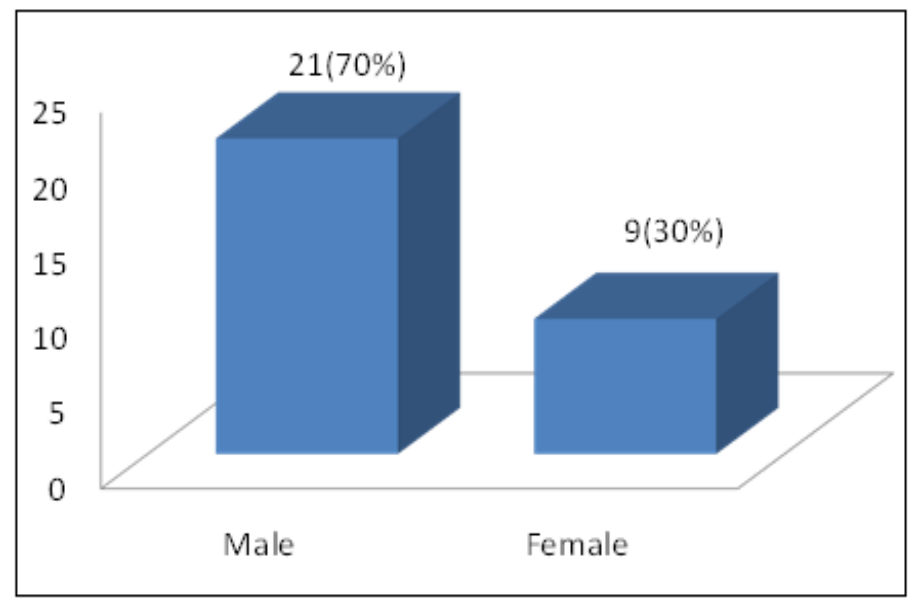

Fig-1: Distribution of patients by sex

Shows distribution of male sex was 21 in number (70\%) and female sex was 9 in number (30\%) (Figure-1).

Table-2: Mode of Injury ( $\mathrm{N}=30)$

\begin{tabular}{|l|l|l|l|}
\hline Sl. No. & Mode of injury & No. of patients & Percentage \\
\hline 01. & Road traffic accident & 21 & 70 \\
\hline 02. & Fall from height & 6 & 20 \\
\hline 03. & Assault & 3 & 10 \\
\hline
\end{tabular}

Distribution of mode of injury occurred RTA in 21 patients $(70 \%)$, fall from height occurred in six cases $(20 \%)$ and assault victim were 3 cases $(10 \%)$ (Table-2).

Table-3: Severity of injury $(\mathrm{N}=30)$

\begin{tabular}{|l|l|l|}
\hline Fracture type & No of Patient & Percentage \\
\hline Stable (minim. displaced) & 9 & 30.0 \\
\hline Unstable(moderately ) (Modera Displaced) & 14 & 46.7 \\
\hline Severely displaced & 7 & 23.3 \\
\hline
\end{tabular}

Stable (minimally displaced) fracture patient were 9 in number $(30 \%)$, unstable (moderately displaced) fracture patient were 14 in number $(46.7 \%)$ and severely displaced patient were seven in number $(23.3 \%)$ (Table-3). 
Distribution of Patients by Level of fracture $(\mathrm{N}=30)$

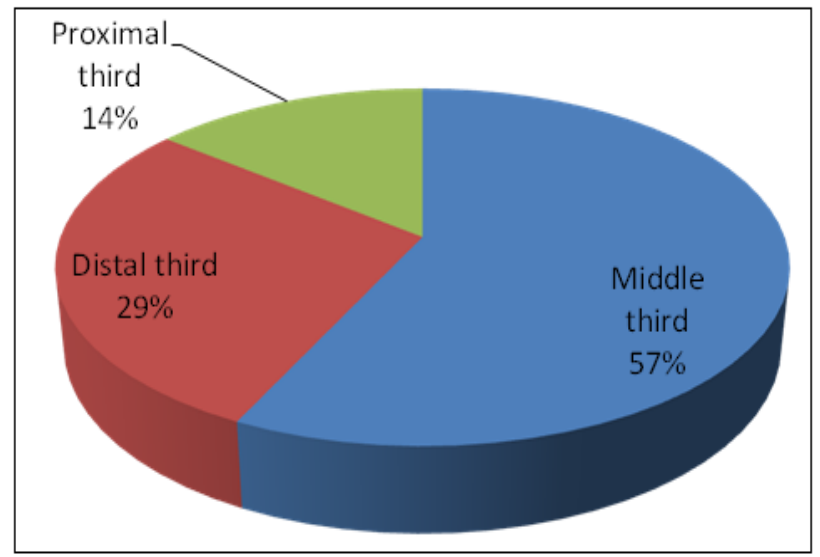

Fig-2: Distribution of Patients by Level of fracture

Figure showed that among 30 patients, middle third fracture was present in $57 \%$, distal third fracture was $29 \%$ and proximal third fracture was $14 \%$ (Figure2).

Table-4: Injury Surgery interval ( $N=30)$

\begin{tabular}{|l|l|l|l|}
\hline Sl. No. & Fixation Time After Trauma & No. of patients & Percentage \\
\hline 1. & $2-7$ days & 12 & $40 \%$ \\
\hline 2. & $8-15$ days & 18 & $60 \%$ \\
\hline
\end{tabular}

Distribution of injury surgery interval: 12 $(40 \%)$ patients were treated within 2-7 days and
$18(60 \%)$ patients were treated within $8-15$ days (Table4).

Table-5: Distribution of Patients by Complication (N=11)

\begin{tabular}{|l|l|l|}
\hline SL. No. & Type of Complication & Number of Patient \\
\hline 1 & Superficial wound infection & 3 \\
\hline 2 & Deep wound infection & 2 \\
\hline 3 & Knee joint movement restriction > 15 degrees & 2 \\
\hline 4 & Knee pain & 13 \\
\hline 5 & Delayed union & 2 \\
\hline
\end{tabular}

Table shows: Superficial wound infection occurred in three cases with moderately displaced-three, deep infection occurred in unstable shaft fracture in two cases, limitation of knee joint movement occurred in two cases, knee pain occurred in thirteen cases and delayed union occurred in two cases (Table-5).

Table-6: Distribution of Patients by Time of the Fracture Union $(\mathrm{N}=30)$

\begin{tabular}{|c|c|c|c|c|c|c|c|}
\hline \multirow[t]{2}{*}{$\begin{array}{l}\text { SI } \\
\text { No }\end{array}$} & \multirow[t]{2}{*}{ Type of fracture } & \multirow[t]{2}{*}{$\begin{array}{l}\text { No. of } \\
\text { Patient }\end{array}$} & $\begin{array}{l}\text { 14-16 } \\
\text { weeks }\end{array}$ & $\begin{array}{l}\text { 16-18 } \\
\text { weeks }\end{array}$ & $\begin{array}{l}18-20 \\
\text { weeks }\end{array}$ & $>20$ weeks & $\begin{array}{l}\text { Mean } \\
\text { weeks }\end{array}$ \\
\hline & & & $\begin{array}{l}\text { No. of } \\
\text { patients }\end{array}$ & $\begin{array}{l}\text { No. of } \\
\text { patients }\end{array}$ & $\begin{array}{l}\text { No. of } \\
\text { patients }\end{array}$ & $\begin{array}{l}\text { No. of } \\
\text { patients }\end{array}$ & \\
\hline 1 & Stable (mini displaced) & 9 & 1 & 1 & 3 & 4 & 18.5 \\
\hline 2 & $\begin{array}{l}\text { Unstable(moderate } \\
\text { displaced) }\end{array}$ & 14 & 6 & 4 & 3 & 1 & \\
\hline 3 & Severely(displaced ) & 7 & 0 & 1 & 3 & 3 & \\
\hline
\end{tabular}

Table Shows: Fracture union occurred early in stable fracture cases and delayed union occurred in cases of severely displaced fracture - one cases, average 18.5 weeks (Table-6). 
Table-7: Final Follow-up findings $(\mathrm{N}=30)$

\begin{tabular}{|l|l|l|l|}
\hline Criteria & Stable(min.) & Unstable (mod.) & Severly displ. \\
\hline & No. of patient & No. of patient & No. of patient \\
\hline Tibial malalignment Varus/Valgus (Degree) & 2 & 1 & 1 \\
\hline Shortening(cm) & 1 & 1 & 2 \\
\hline Range of knee motion & & & \\
\hline Flexion (Degree) & 11 & 5 & 4 \\
\hline Extension deficits (Degree) & 1 & 2 & 3 \\
\hline Range of ankle motion & & & \\
\hline Dorsi-flexion (Degree) & 11 & 4 & 2 \\
\hline Planter-flexion (Degree) & 9 & 3 & 1 \\
\hline Foot motion (as compared to normal) & 11 & 6 & 3 \\
\hline Pain in the Limb & 3 & 2 & 3 \\
\hline
\end{tabular}

Table shows distribution of follow up findings good knee movement and ankle movement (Table-7).

Distribution of Patient by final outcome

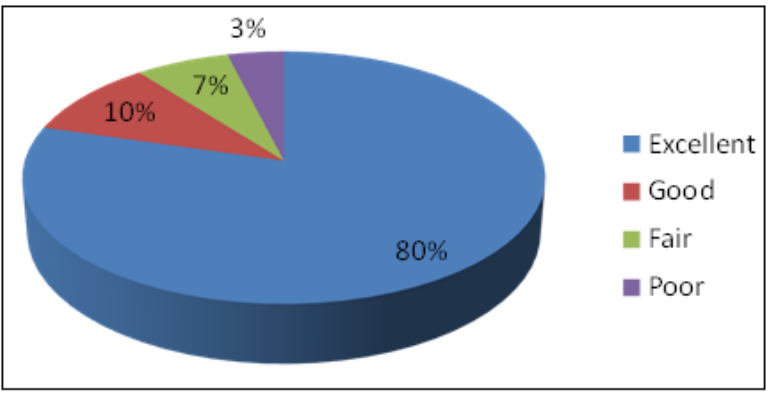

Fig-3: Pie chart of patient by the final outcome

Figure shows the rating of final outcome of closed interlocking intramedullary nailing after thirtysix weeks follow-up period. Among 30 patients, 24 (80\%) were excellent, $3(10 \%)$ were good, 2 (7\%) were fair and $1(3 \%)$ was poor (Figure-3).

\section{Final Outcome Categorization}

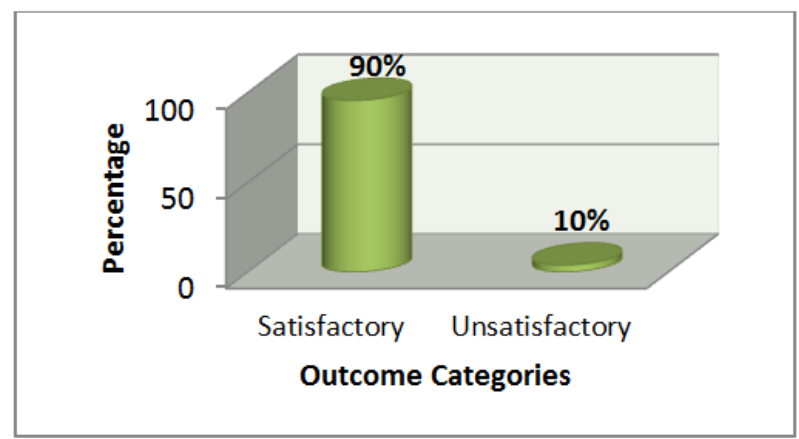

Fig-4: Distribution of patients by the Final Outcome Categorization

Figure shows that among 30, the final outcome were satisfactory in $27(90 \%)$ cases and Unsatisfactory were in $3(10 \%)$ cases [Figure 4].

Table-8: Comparison between planter flexion Dorsiflexion of ankle $(n=30)$.

\begin{tabular}{|l|l|l|l|}
\hline Outcome & Healthy limb Mean \pm SD & Injured limb Mean \pm SD & P-value \\
\hline Planter flexion of ankle & $39.87 \pm 0.507$ & $36.07 \pm 1.929$ & $\mathrm{P}>.05$ \\
\hline Dorsiflexion of ankle & $24.87 \pm 0.434$ & $21.57 \pm 2.788$ & $\mathrm{P}>.05$ \\
\hline
\end{tabular}

Planter flexion and dorsiflexion of ankle of injured and healthy sides were measured at the last follow up. T-test was done for calculation of test statistic and we found that there were no significant difference between planter flexion of the healthy and injured limb after treatment (Table-8).

\section{DISCUSSION}

Closed fractures shaft of the tibia are one of the common injuries attending in casualty department of DMCH and NITOR. The optimal treatment of tibial shaft fractures remains controversial. In the review of 705 such fractures [7], stated that "fractures of the tibia are important for two reasons: 1-they are common, 2they are controversial, and anything that is both common and controversial must be important "A stable isolated closed fracture of the tibial shaft can be adequately managed by closed reduction and immobilization with a plaster cast or brace, but there are indications for closed nailing. Certain fracture patterns are difficult to immobilize with plaster cast or brace. They include fractures in the distal third of the tibia that may shorten or angulate into an acceptable position as well as unstable, comminuted or segmental fracture with potential for nonunion [8]. The prognosis of closed fracture tibia depends largely on the mechanism of injury, fracture configuration, degree of soft tissue injuries and time elapsed since the injuries. Teneous blood supply of the tibia and thin soft tissue coverage make the tibia than for fractures of the femur [8]. This prospective observational study has been undertaken in DMCH, Dhaka from a period between July 2011 to December 2012 and titled as "Evaluation 
of the outcome of treatment of tibial shaft fracture by close reduction and internal fixation with interlocking intramedullary nail "30 cases were included in the study. In this series an attempt has been made to perform nailing within 5 days after admission in fresh injury to minimize swelling and soft tissue healing by elevation of limb and long leg back slab. In this series any fracture pattern in the tibial diaphysis from $10 \mathrm{~cm}$ below knee and $5 \mathrm{~cm}$ above the ankle was fixed by $\mathrm{AO}$ interlocking intramedullary tibial nail. According to Grosse Kempf, interlocking nail can be applied in any fracture if at least 3 to $4 \mathrm{~cm}$ of bone is intact proximally and distally to the fracture site [9]. We have shown that a series of policy of closed nailing in all displaced closed tibial shaft fractures, can give good results with a relatively low complication rate. Interlocking intramedullary nail of the tibia has proved to be an excellent method of treatment. Knee pain seems to be the main problem associated with the technique [10], and generally experienced with kneeling. In the present series knee pain on kneeling was in 13 cases $(44.44 \%)$. This was comparable with the $40.80 \%$ of [10], study and become painless after removal of the nail. In this series, maximum age incidence were in 25-34 year age group $(53.3 \%)$ and second highest were 18 -24 year group (20\%). Range was $18-55$ years with mean age 31.8 year. In the study of [8], showed that tibial fracture occurs most in 31 year age group ( range $14-77$ ). In the study of [10], also showed most of victims were in 32.4 years ( range 16 to 80 ). These group represent, the most young age group having high activity for earning who needs an ambulant life and early return to job or activity. Closed tibial shaft fractures were found more in male than female. In present series 21 cases $(70 \%)$ were male and 9 cases $(30 \%)$ were female. Male female ratio (3: 1). In [8], study 90 male and 30 female which are similar to present series .In the study of [10]. 100 were male and 30 were female; male female ratio approximately (3: 1). This may be due to fact that female are less exposed to road traffic accidents in this country because of their frequency of traveling is considerably less than that of male. Road traffic accidents were found to be the most common cause of injury in this series -21 cases $(70 \%)$ out of 30 . In [8], study $90 \%$ injury due to road traffic accidents. In [10], study $31.2 \%$ fractures followed road traffic accidents, $36 \%$ followed sports and $28 \%$ occurred after fall from height or stairs. In the study of [11], $89 \%$ fractures were sustained in road traffic accident and $10 \%$ in sports accident .In this study most fracture occurred in middle third (57\%) followed by distal third (29\%), In [8], study most fracture were in the middle third of the tibial shaft. In the [10], $51 \%$ fractures were in distal third, $37 \%$ fractures were in middle third. In [11], study $50 \%$ fractures were in middle third, $37 \%$ in the distal third and $13 \%$ were in proximal third. In present series initial displacement of fracture shaft were mild $(<50 \%)$ in $46.7 \%$ cases followed by moderate (50 to $100 \%$ ) in $30 \%$ cases and severe displacement $(>100 \%)$ in 23.3
$\%$ cases depending on percent of bone diameter. In the study of [12], mild displacement were in $62 \%$, moderate $28 \%$ and severe in $10 \%$ cases .Hospital stay is one of the important part of this study . Minimum hospital stay was 2 days and maximum 15 days in fresh cases with a mean stay of 5 days .In study [10], mean hospital stay was 7.1 days [9], study showed mean hospital stay was 9.8 days. Conservative management associated with a prolonged hospitalization period if traction was used. The reported time vary between 10 days [13].The average times in hospital following AO plating is 12 to 15 days [14]. In our study hospital stay was less in primarily treated fracture was average 5 days. In this series 3 cases needed prolonged hospital stay because of medical problems. In this series, time of union was ranging from 14 to 36 weeks and with a mean of 18.5 weeks. In the study of [10], mean time of union was 16.7 weeks and 12 weeks in series [9]. Showed a mean time of union was 22.5 weeks [15]. Following AO plating, time of union was 16.4 to 19.4 weeks depending upon the type of closed fracture in the study [16]. The mean operating time in this series was 65 minutes (range 50 to 140). The variation in time mainly being due to technical difficulties like inserting the distal screw; closed reduction with $\mathrm{C}$-arm and some fractures close reduction and internal fixation. In study the mean operating time was 62 minutes (range 25 to 160) [10]. In the study of [11], the mean operating times was 38 minutes (range 20 -120). In this series partial weight-bearing was advised in 24 (72.22\% 0cases by six weeks and 4 cases $(27.78 \%)$ cases by 12 weeks. Advised weight bearing by six weeks in $92.8 \%$ cases. In the present series $17(55.5 \%)$ had full movement in the knee and ankle by three months, 9 $(27.4 \%)$ by six months and $4(11.1 \%)$ by 9 months [10]. In the $65.6 \%$ patients have full movement in the knee and ankle by three months, $24 \%$ by six months and $3.2 \%$ by one year [10]. Out of 30 cases , 3 ( $3 \%$ ) cases had superficial wound infection at the site .Wound swab yield the growth of mixed bacteria which were resistant to almost all usual antibiotic such as penicillin ,amoxicillin ,cloxacillin , cotrimoxazole and tetracycline .They were only sensitive to ciprofloxacine, ceftazimidine and nitriamycin. Skin infections were managed by flashing with normal saline followed by povidone iodine solution dressing. Ceftazimidine was given according to culture sensitivity for four weeks and wound healed. In the study of [11], superficial infection rate was $3.5 \%$. Two cases were deep infection in this series. One occurred eight months after operation and presented with infrapatellar abscess which do not communicate with joint. Abscess was drained and antibiotic was given according to culture and sensitivity for six weeks but wound was not healed. Radiologically bony union was achieved but no sign of osteomyelitis was found. So nail was removed and antibiotic was given according to culture and sensitivity for six weeks and wound was healed. The second deep infection occurred after eleven months of operation and presented 
with increasing pain and swelling at the proximal locking screw. Radiograph showed bony union with sign of infection but no osteomyelitis then nail was removed, pus was found at proximal locking screw and irrigation by normal saline and antibiotic given according to culture and sensitivity for six weeks, after the wound was healed. Deep infection rate was $1.6 \%$ in [10] study and $3 \%$ in series [17]. In this series two patients had flexion deficit at knee joint of 45 degree 25 degree and deficit of ankle motion more than 10 degree in these cases. In series and study flexion deficit of knee joint 35 degree in 4 cases $(8.88 \%)$ and ankle motion were deficit more than 10 degree of same 4 cases $[6,10$, $18]$.

\section{Conclusion}

The present study was undertaken to investigate the outcome of closed interlocking nailing of diaphyseal fractures of tibia, done with the help of image intensifier. 30 cases of closed tibial diaphyseal fractures were fixed with intramedullary interlocking nail. The cases were followed up minimum for a period of nine months. The fractures in our study united in an average of about 18.5 weeks. Interlocked intramedullary nailing done under image intensifier has proved to be a one-time procedure leading to union in almost all the cases. This procedure allows earlier weight bearing leading to earlier fracture union with less morbidity. Because of the high union rate and low infection rate, we consider closed interlocking nailing as the best mode of treatment for diaphyseal tibial fractures and early return to function.

\section{REFERENCES}

1. Russel, T. A. Fractures of the tibia and fibula. In Rockwood and Greens fracture in adults. $4^{\text {th }} \mathrm{ed}$. New-York; 2132-6.

2. Charnley, J. (1977). The closed treatment of common fractures. Edinburgh: Churchill Livingston. 23-30.

3. Hindley, C. J. (1988). Closed medullary nailing for recent fractures of the tibia, Injury, 19, 180-4.

4. Denham, R. A., Johnson, E. E., \& Marder, R. (1964). Open intramedullary nailing and bone grafting for non -union of tibial diaphyseal fracture. Journal Bone Joint Surgery Am, 69(3):375-380.

5. Watson, J. T. (2002). Treatment of unstable fractures of shaft of the tibia. Journal Bone Joint Surgery, 76-(A), 1575-1584.

6. Alho, A., Ekeland, A., Stromsoe, K., Folleras, G., \& Thoresen, B. O. (1990). Locked intramedullary nailing for displaced tibial shaft fractures. Journal Bones Joint Surgery (Br), 72, 805-809.

7. Nicoll, E. A. (1964). Fracture of the tibal shaft' a study 705 cases. Journal Bone joint Surgery (Br), 46-B (3):373-387.

8. Bone, L. B., \& Johnson, K. D. (1986). Treatment of tibial fractures by reaming and intramedullary nailing. Journal Bone Joint Surgery (Am), 68(6):877-887.

9. Pintore, E., Maffulli, N., \& Petricciuolo, F. (1992). Interlocking nailing for fracture of femur and tibia. Injury, 23(6):381-6.

10. Court-Brown, C. M. Christie, J., \& McQueen, M. M. (1990). Closed intramedullary tibial nailing: its use in closed and type 1 open fractures. Journal Bone Joint Surgery (Br), 72B, 605-611.

11. Smet, K. D., Mosert, A. K., Witte, J. D., Brauwer, V., De, Verdonk, R. (2000). Closed intramedullary tibial nailing using the Marchetti-Vicenzl nail. Injury, 31(8):597-603.

12. Blachut, P. A., O, Brein. P. J., Meek R. N., \& Broekhuyse, H. M. (1997). Interlocking intramedullary nailing with or without reaming for the treatment of closed fracture of the tibial shaft. Journal Bone Joint Surgery (Br.), 79:640-646.

13. Kay, L., Hansen, B. A., \& Raaschou, H. O. (1986). Fractures of the tibial shaft conservatively treated. Injury, 17:5-11.

14. Thunold, J., Varhaug, J. E., \& Bjerkest, T. (1975). Tibial shaft fractures treated by rigid internal fixation: The early results in a 4 years series. Injury, 76(7):12-133.

15. Anglen, J. O., \& Blue, J. M. (1995). A Comparison of reamed and undreamed nailing of the tibia. Journal Trauma, 39(2):351-355.

16. Batten, R. L., Donaldson, L. J., \& Aldridge, M. J. (1978). Experience with the AO method in the treatment of 142 cases of fresh fractures of the tibial shaft treated in the UK. Injury, 10:108-114.

17. Wiss, D. A., \& Stetson, W. B. (1995). Unstable fractures of the tibia treated with a reamed intramedullary interlocking nail. Clin Orthop, 315:56-63.

18. Folleras, G., Alho, A., Stromsoe, K., Ekeland, E., \& Thoresen, B. O. (1990). Locked intramedullary nailing of fractures of femur and tibia. Injury, 21(6):385-388.

19. Edwards, C. C., Simmons, S. C., Browner, B. D., \& Weigel, M. C. (1988). Severe open tibial fractures. Results treating 202 injuries with external fixation. Clinical orthopaedics and related research, (230), 98-115. 